\title{
Wolbachia variant that induces two distinct reproductive phenotypes in different hosts
}

\author{
T Sasaki ${ }^{1}, \mathrm{~N}$ Massaki $^{2}$ and T Kubo ${ }^{2}$ \\ ${ }^{1}$ Honeybee Science Research Center, Tamagawa University, Machida, Tokyo 194-8610, Japan; ${ }^{2}$ Department of Biological Sciences, \\ Graduate School of Science, University of Tokyo, Hongo, Tokyo 113-0033, Japan
}

\begin{abstract}
Wolbachia is an intracellular endosymbiont that induces a variety of reproductive alterations in diverse arthropods. The almond moth, Cadra cautella, is double infected with two Wolbachia variants, $w C a u A$ and $w C a u B$, and expresses complete cytoplasmic incompatibility $(\mathrm{Cl})$. The individual contribution of $w C a u A$ and $w C a u B$ to the expression of $\mathrm{Cl}$ are unclear, however, because the two variants have not been separated in this host. The effect of $w C a u A$ is of particular interest because it induces male killing when transferred into the Mediterranean flour moth, Ephestia kuehniella. In the present study, we generated $C$. cautella infected with only wCauA by treating double-infected insects
\end{abstract}

with tetracycline. Single-infected $C$. cautella exhibited strong $\mathrm{Cl}$, demonstrating that wCauA induces two distinct reproductive phenotypes in different hosts: $\mathrm{Cl}$ in $C$. cautella and male killing in E. kuehniella. $\mathrm{Cl}$ was also observed in the cross of double-infected males and single-infected females. Comparison of the single- and double-infected insects by real-time quantitative polymerase chain reaction suggested that the $w$ CauA density is not affected much by the presence or absence of $w C a u B$.

Heredity (2005) 95, 389-393. doi:10.1038/sj.hdy.6800737; published online 17 August 2005

Keywords: Wolbachia; Cadra cautella; cytoplasmic incompatibility; male killing; double infection; real-time quantitative PCR

\section{Introduction}

Wolbachia is a maternally inherited intracellular symbiont that is widespread in arthropods (Bouchon et al, 1998; Jeyaprakash and Hoy, 2000) and filarial nematodes (Bandi et al, 1998). In its arthropod hosts, Wolbachia induces a variety of reproductive alterations such as cytoplasmic incompatibility (CI), thelytokous parthenogenesis, male killing, and feminization (reviewed in Werren, 1997; Bourtzis and Braig, 1999; Stouthamer et al, 1999).

There are two types of CI: unidirectional and bidirectional. Unidirectional CI typically occurs when infected males mate with uninfected females, whereas the crosses of other combinations are compatible. This phenomenon is generally explained by 'modification' of the sperm and 'rescue' in the infected eggs (Werren, 1997). Unidirectional CI decreases the reproductive success of uninfected females, and promotes the spread of infection (Turelli and Hoffmann, 1991). Bidirectional incompatibility often occurs when males and females are infected with different variants of Wolbachia, and is explained by variations in the 'modification' and 'rescue' functions of the different Wolbachia variants (O'Neill and Karr, 1990; Rousset and De Stordeur, 1994).

The variety of reproductive alterations induced by Wolbachia raises the question of how different phenotypes arise. Some experimental transfers of Wolbachia between different host species revealed that Wolbachia

Correspondence: T Sasaki, Honeybee Science Research Center, Tamagawa University, 6-1-1 Tamagawa Gakuenn, Machida, Tokyo 194-8610, Japan. E-mail: tsasaki@lab.tamagawa.ac.jp

Received 19 November 2004; accepted 22 July 2005; published online 17 August 2005 induces similar phenotypes in the recipient host as in the donor host, but host differences might affect the penetrance of the phenotype (eg, Boyle et al, 1993; Clancy and Hoffmann, 1997; Poinsot et al, 1998). Transfers of feminizing Wolbachia among isopods resulted in four possible outcomes: no reproductive effect, expression of feminization, death of recipients, and failure of transinfection (Bouchon et al, 1998). Thus, the reproductive phenotypes appear to result from a complex interaction of host and bacterial components.

The almond moth, Cadra cautella, is double infected with $w \mathrm{CauA}$ and $w \mathrm{CauB}$, which belong to the A- and B-Wolbachia groups designated by Werren et al (1995), respectively, and expresses complete CI (Sasaki and Ishikawa, 1999). When the Wolbachia were transferred from C. cautella into the Mediterranean flour moth, Ephestia kuehniella, in which wCauA and wCauB were segregated, wCauA induced male killing in the new host (Sasaki et al, 2002). This suggests that wCauA induces different phenotypes in the two host insects. The effect of $w$ CauA on its natural host C. cautella, however, is not clear because the host is double infected, and singleinfected insects have not been obtained.

In this study, we generated a single-infected C. cautella strain carrying only wCauA by treating the doubleinfected insects with tetracycline. The primary aim was to examine the phenotype expressed in the singleinfected insects. Crossing experiments demonstrated that wCauA alone induces strong CI. We also investigated two aspects of double infection using single- and double-infected C. cautella strains. First, we examined the crossability of the two strains, and observed that doubleinfected males were incompatible with single-infected females. Second, we used real-time polymerase chain 
reaction (PCR) to measure the density of Wolbachia in ovaries, in which no significant difference in the density of $w$ CauA was found between the two lines. These results are discussed in conjunction with how double infection is maintained.

\section{Materials and methods}

\section{Insects}

C. cautella, which was double infected, was originally collected in Tsuchiura, Japan. The uninfected strain was generated by tetracycline treatment (Sasaki and Ishikawa, 1999). The insects were reared on a diet consisting of wheat bran, dried yeast, and glycerol (20:1:2) at $25^{\circ} \mathrm{C}$ under a $16 \mathrm{~h}$ light: $8 \mathrm{~h}$ dark photoperiod.

\section{Tetracycline treatment}

One approach to obtain wCauA-single-infected C. cautella is to transfer wCauA back into uninfected C. cautella from the transinfected E. kuehniella bearing only this Wolbachia variant, but artificial transfer of Wolbachia into C. cautella has not been successful. Therefore, we attempted to use antibiotic treatment to remove $w$ CauB from doubleinfected C. cautella.

The duration of $C$. cautella larval development at $25^{\circ} \mathrm{C}$ is approximately 3 weeks. To obtain single-infected lines, the double-infected insects were reared on a diet containing $0.04 \%$ tetracycline for the first week during larval development. Approximately 200 eggs were placed on $10 \mathrm{~g}$ of the diet with tetracycline so that the larvae were fed the diet as soon as they hatched. One week after hatching, $200 \mathrm{~g}$ of the diet without tetracycline was added to the rearing case and mixed gently with the tetracycline diet on which the larvae were growing. The insects treated with tetracycline were referred to as generation 0 (G0).

G1 eggs were collected from individual G0 females, and diagnosed for infection by PCR (see below). G1 and subsequent generations were reared without antibiotics. Selection for single-infected lines was continued for four generations.

\section{Diagnostic PCR for infection}

The template DNA was prepared from eggs or adults according to O'Neill et al (1992). Each egg was homogenized in $15 \mu \mathrm{l}$ STE $(100 \mathrm{mM} \mathrm{NaCl}, 10 \mathrm{mM}$ Tris- $\mathrm{HCl}$ $1 \mathrm{mM}$ EDTA, pH 8.0) containing $0.4 \mathrm{mg} / \mathrm{ml}$ proteinase $\mathrm{K}$, and incubated for $90 \mathrm{~min}$ at $55^{\circ} \mathrm{C}$ and then for $15 \mathrm{~min}$ at $95^{\circ} \mathrm{C}$. When DNA was extracted from adults, each individual was first homogenized in $100 \mu$ STE without proteinase $\mathrm{K}$, and $10 \mu \mathrm{l}$ of the homogenate was added to $90 \mu \mathrm{l}$ STE containing proteinase $\mathrm{K}$.

To test the infection status, two primer sets for the ftsZ bacterial cell-cycle gene were used (Werren et al, 1995). The A group-specific ftsZ primers were ftsZAdf (5'-CTC AAG CAC TAG AAA AGT CG-3') and ftsZAdr (5'-TTA GCT CCT TCG CTT ACC TG-3'), and the B groupspecific primers were ftsZBf $\left(5^{\prime}-\mathrm{CCG}\right.$ ATG CTC AAG CGT TAG AG-3') and ftsZBr (5'-CCA CTT AAC TCT TTC GTT TG-3'). PCR was performed in a $20-\mu 1$ reaction mixture using Takara EX Taq. The cycling conditions were $94^{\circ} \mathrm{C}$ for $3 \mathrm{~min}$ followed by 35 amplification cycles of $94^{\circ} \mathrm{C}$ for $30 \mathrm{~s}, 55^{\circ} \mathrm{C}$ for $30 \mathrm{~s}$, and $72^{\circ} \mathrm{C}$ for $1 \mathrm{~min}$, and finally $72^{\circ} \mathrm{C}$ for $5 \mathrm{~min}$.

\section{Incompatibility test}

Crosses were performed using single pairs of virgin individuals. Since the male larva is easily distinguished by a dark patch (the testes) on its back, we separated the females and males at the late larval stage to obtain virgin moths. A female and a male, less than 2 days after emergence, were put in a plastic cup $(3 \mathrm{~cm}$ in diameter, $5 \mathrm{~cm}$ in height) and left there for 3 days. Successful mating was judged from the number of eggs deposited, as unmated females produce few eggs. From the mating cups in which more than 50 eggs were found, 50-100 eggs were collected and placed onto $1 \%$ agarose in a plastic dish ( $35 \mathrm{~mm}$ in diameter). After incubation for $7-8$ days at $25^{\circ} \mathrm{C}$, the hatching rate was scored for each single-pair cross.

\section{Real-time quantitative PCR}

DNA was extracted from ovaries of individual females by phenol extraction followed by ethanol precipitation, as previously described (Ikeda et al, 2003a). Quantitative PCR was performed in a LightCycler (Roche Diagnostics) using variant-specific primers and probes for the groEL gene according to Ikeda et al (2003b). The groEL of wCauA was amplified using the primers groEL415AF $\left(5^{\prime}-\right.$ CAA TGT CTC GTA CAA TTT CTC-3') and groEL641AR (5'-GAT TAT CAA GCT CCA CGA-3'). The PCR product was detected with the probes groELA1 (5'-AAA GAG TTA GAA GTT GAG CTG ACT ACT GG-3'-FITC) and groELA2 (LC-Red 640-5'-ATG CAA TTT GAT CGC GGT TAT CTC TCT CCG-3'). The groEL of $w$ CauB was amplified with the primers groEL415BF (5'-CAA TGT CTC GCA CAA TTT CTT-3') and groEL641BR (5'-GAT CAT CAA GCT CCA CGC-3'), and the product was detected with the probes groELB1 (5'-GAA GTT GAA CTT ACA ACT GGT ATG CAG TT-3'-FITC) and groELB2 (LC-Red 640-5'-GAC CGT GGT TAT CTC TCT CCA TAC $\left.-3^{\prime}\right)$.

The $10-\mu \mathrm{l}$ reaction mixture consisted of $1 \mu \mathrm{l}$ LightCycler Master Mixture (Roche Diagnostics), $30 \mathrm{nmol}$ $\mathrm{MgCl}_{2}, 5$ pmol each primer, 2 pmol probe 1, 4 pmol probe 2 , and $1 \mu \mathrm{l}$ template DNA. The PCR cycling conditions were $30 \mathrm{~s}$ at $95^{\circ} \mathrm{C}$ followed by 45 cycles of $1 \mathrm{~s}$ at $95^{\circ} \mathrm{C}, 10 \mathrm{~s}$ at $52^{\circ} \mathrm{C}$, and $30 \mathrm{~s}$ at $72^{\circ} \mathrm{C}$.

Standard DNAs were prepared from PCR products amplified from $w \mathrm{CauA}$ and $w$ CauB using the primers groEL49F (5'-GTT GCA AGA AGC CTT TCG TG-3') and groEL827R (5'-CCA AAA CCT GGA GCT TTT ACT G$\left.3^{\prime}\right)$. The PCR products were electrophoresed on $1.0 \%$ agarose gel, extracted from the gel using QIAquick Gel Extraction Kit (Qiagen), and quantified based on the optic absorbance at $260 \mathrm{~nm}$. Dilutions (10-fold) from $10 \mathrm{pg} / \mu \mathrm{l}$ to $1 \mathrm{fg} / \mu \mathrm{l}$ were used to draw standard curves, in which amplification kinetics showed a linear relationship with the concentration of template DNA. We also confirmed that the amplification efficiency of the standards is not affected by the addition of the DNA from the ovaries of uninfected females extracted in the same way as the sample DNA, so the presence of any PCR inhibitors in the sample is negligible.

\section{Results}

\section{Selection of wCauA-single-infected lines}

G1 eggs were collected from 28 females that were treated with tetracycline during the first week of the larval 
period. Infection was examined by testing two eggs from each brood. Of the 56 eggs examined, 20 were $w$ CauA positive and $w \mathrm{CauB}$ negative, two were $w \mathrm{CauA}$ negative and $w$ CauB positive, 29 were double positive, and five were double negative. The broods in which at least one wCauA-positive and wCauB-negative egg was found were maintained for further selection of $w$ CauA-singleinfected lines. Selection was performed for three subsequent generations and nine single-infected lines were obtained at G4. When G5 eggs were collected from 20 females, the females were diagnosed after oviposition. We confirmed that all mothers were infected with $w$ CauA, but not with $w \mathrm{CauB}$, and the G5 eggs were pooled as the wCauA-single-infected strain.

We also attempted to obtain wCauB-single-infected lines from the G1 broods in which wCauA-negative and $w$ CauB-positive eggs were found. All 30 eggs examined at the G2 generation, however, were double positive, and we did not obtain wCauB-single-infected lines.

\section{Incompatibility}

Crosses were performed using the wCauA-singleinfected strain at generations 9 and 10. Prior to the experiments, infections of the single-infected stain at generation 9 and double-infected strain were examined by testing 46 eggs selected at random for each strain. All eggs from the single-infected stain were positive for $w$ CauA and negative for $w \mathrm{CauB}$, and all eggs from the double-infected strain were positive for both Wolbachia variants, suggesting that the transmission of Wolbachia is very stable.

Incompatibility assays are shown in Table 1 . Since the data had skewed distributions, differences in mortality rate among treatments were assessed using nonparametric Mann-Whitney $U$-test. Normal approximation was used in comparisons including a sample whose size was greater than 20. Single-infected males are incompatible with uninfected females ( $\mathrm{A}$ vs $\mathrm{B}, U=288, P<0.001$ ), indicating that wCauA alone induces $\mathrm{CI}$ in $\mathrm{C}$. cautella. The incompatibility was almost complete: of the 16 crosses, no eggs hatched in 14 crosses and only one egg hatched in each of the other two crosses.

Unidirectional incompatibility also occurred between single-infected and double-infected strains ( $\mathrm{F}$ vs $\mathrm{H}$, $Z=5.38, P<0.001)$. In addition, there was a slight but significant increase in mortality in the cross of doubleinfected individuals, compared with crosses between uninfected males and single- or double-infected females (I vs $\mathrm{D}, \mathrm{Z}=2.59, P<0.01 ; \mathrm{I}$ vs $\mathrm{G}, \mathrm{Z}=3.24, P<0.01$ ).
Wolbachia densities in single- and double-infected C. cautella

Wolbachia density in ovaries was measured by real-time quantitative PCR when the single-infected strain was at G11 (Table 2). The data were normalized with the tissue mass rather than with a host gene as an internal control, because most Wolbachia were expected to be in ova while most host's DNAs originated in the cells of ovarian tubes surrounding the ova. There was no significant difference in wCauA densities between the single- and doubleinfected strains (Student's $t=0.94, P>0.2$ ).

In the double-infected strain, the density of wCauA was higher than that of $w \mathrm{CauB}(t=4.66, P<0.001)$. This difference in density might, in part, be the reason for the difficulty in obtaining $w \mathrm{CauB}$-single-infected lines.

\section{Discussion}

We demonstrated that $w$ CauA induces $\mathrm{CI}$ in C. cautella (Table 1). wCauA induces male killing in E. kuehniella (Sasaki et al, 2002). Thus, wCauA includes two distinct types of host-dependent reproductive alterations.

There are at least two possible explanations for the induction of two different phenotypes by wCauA. One is that $w$ CauA has different genes to induce CI and male killing. C. cautella, but not E. kuehniella, might be resistant to the action of the male-killing gene, or the gene expression is suppressed exclusively in $C$. cautella. The second explanation is that the same gene is responsible for the expression of the two phenotypes and the same bacterial action induces different responses in the hosts. $\mathrm{CI}$ is believed to result from sperm modification by Wolbachia in the testis, whereas male killing occurs due to the presence of Wolbachia in eggs. Therefore, wCauA might affect male-specific gene expression mechanisms common to spermatogenesis and male embryonic

Table 2 Infection density of Wolbachia in Cadra cautella ovaries

\begin{tabular}{llcc}
\hline Host & Wolbachia & $\begin{array}{c}\text { groEL copies } \\
\left(\times 10^{7}\right) / \text { ovary }\end{array}$ & $\begin{array}{c}\text { groEL copies } \\
\left(\times 10^{7}\right) / m g \text { ovary }\end{array}$ \\
\hline Single infected & $w$ CauA & $5.28 \pm 1.49$ & $1.03 \pm 0.26$ \\
Double infected & $w$ CauA & $6.34 \pm 1.50$ & $1.12 \pm 0.23$ \\
& $w$ CauB & $4.36 \pm 0.71$ & $0.78 \pm 0.12$ \\
\hline
\end{tabular}

Ovaries were collected from females $60-72 \mathrm{~h}$ after adult emergence. The Wolbachia population was expressed in terms of groEL gene copy number. The mean $\pm \mathrm{SD}(n=12)$ is given for each Wolbachia strain

Table 1 Mortality rates in crosses between Cadra cautella strains

\begin{tabular}{|c|c|c|c|c|c|}
\hline \multirow[t]{2}{*}{ Cross } & \multirow[t]{2}{*}{ Female } & \multirow[t]{2}{*}{ Male } & \multirow[t]{2}{*}{ No. of pairs } & \multicolumn{2}{|c|}{ Mortality (\%) } \\
\hline & & & & Median & Interquartile range \\
\hline A & Tet & Tet & 18 & 14.8 & [9.9-30.3] \\
\hline B & Tet & $w$ CauA & 16 & 100.0 & [100.0-100.0] \\
\hline $\mathrm{C}$ & Tet & $w \mathrm{CauA}+w \mathrm{CauB}$ & 19 & 100.0 & [100.0-100.0] \\
\hline $\mathrm{D}$ & $w \mathrm{CauA}$ & Tet & 15 & 11.1 & [8.1-19.2] \\
\hline $\mathrm{E}$ & $w \mathrm{CauA}$ & $w$ CauA & 22 & 21.2 & [8.4-24.8] \\
\hline $\mathrm{F}$ & $w \mathrm{CauA}$ & $w \mathrm{Cau} \mathrm{A}+w \mathrm{CauB}$ & 26 & 94.0 & [87.7-94.3] \\
\hline G & $w \mathrm{Cau} \mathrm{A}+w \mathrm{CauB}$ & Tet & 14 & 10.0 & [8.3-13.0] \\
\hline $\mathrm{H}$ & $w \mathrm{Cau} \mathrm{A}+w \mathrm{CauB}$ & $w$ CauA & 16 & 21.3 & [7.5-28.1] \\
\hline I & $w \mathrm{Cau} \mathrm{A}+w \mathrm{CauB}$ & $w \mathrm{Cau} \mathrm{A}+w \mathrm{CauB}$ & 23 & 23.0 & [13.9-24.6] \\
\hline
\end{tabular}


development. Different phenotypes could be expressed if the male-specific gene expression mechanisms are similar but not the same in the two host insects.

We assume that the second possibility is more likely, because if $w$ CauA has different genes to induce $\mathrm{CI}$ and male killing as in the first explanation, it is difficult to explain how the gene for male killing is maintained in $w$ CauA that does not express this phenotype in its natural host. The presence of a common mechanism for CI and male killing has also been suggested in Drosophila bifasciata, in which a change of phenotype from male killing to CI was observed (Hurst et al, 2000). The authors reported that the females infected with male-killing Wolbachia laid eggs with decreased bacterial density when they were exposed to elevated temperatures, and the male offspring produced following the high temperature treatment exhibited weak CI.

Two other significant observations in the present study are that unidirectional incompatibility occurs between single- and double-infected strains, and that the density of $w$ CauA does not differ significantly between the two strains. These observations provide insight into the mechanism of how double infection is maintained.

There are double infections of Wolbachia in many species (eg, Werren et al, 1995; Jeyaprakash and Hoy, 2000; Kittayapong et al, 2003). It has been shown that double-infected males are incompatible with singleinfected females in some insects such as $D$. simulans (Merçot et al, 1995; Rousset and Solignac, 1995), Aedes albopictus (Sinkins et al, 1995), and Nasonia vitripennis (Perrot-Minnot et al, 1996). This incompatibility has been explained by the specific interaction between sperm modification and rescue as follows: each Wolbachia variant modifies the sperm in its particular way and the sperm modified by two different Wolbachia variants cannot be rescued in single-infected eggs. This incompatibility is considered to be the mechanism by which double infection spreads and is maintained in a host population. Therefore, it is expected that incompatibility between double- and single-infected individuals is common in double-infected hosts. Our observation in C. cautella provides additional evidence to support this view.

There was a slight increase in mortality in the cross of double-infected males and females. Such mortality within multiple-infected insects is also observed in $D$. simulans, and is explained by an incomplete rescue effect that possibly occurs due to an unequal transmission of some of the Wolbachia variants to the embryos (Rousset et al, 1999).

Besides incompatibility, the maternal transmission rate of Wolbachia is a critical factor that determines the spread and maintenance of infection, and the transmission is more or less affected by the density of Wolbachia. To understand the transmission of double infection, it is important to clarify how the density of the two different Wolbachia variants is regulated. There might be an interaction such as competition between the two Wolbachia variants. Alternatively, proliferation of the two Wolbachia variants might be independent of each other. Our data from quantitative PCR suggest that there is no strong competitive interaction between $w \mathrm{CauA}$ and $w \mathrm{CauB}$ in the double infection. This is in agreement with previous studies of two transinfected insects, $D$. simulans (Rousset et al, 1999) and E. kuehniella (Ikeda et al, 2003a, b), and naturally infected Leptopilina heterotoma (Mouton et al, 2003). Independent regulation does not decrease the density of each Wolbachia variant, and therefore appears to be suitable for stable transmission of double infection.

\section{Acknowledgements}

We thank Dr S Hoshizaki for comments on the manuscript. This work was supported by a Grant-in-Aid for Scientific Research (grant no. 14740461) from the Japan Society for the Promotion of Science.

\section{References}

Bandi C, Anderson TJ, Genchi C, Blaxter ML (1998). Phylogeny of Wolbachia in filarial nematodes. Proc $R$ Soc Lond $B$ 265: 2407-2413.

Bouchon D, Rigaud T, Juchault P (1998). Evidence for widespread Wolbachia infection in isopod crustaceans: molecular identification and host feminization. Proc $R$ Soc Lond B 265: 1081-1090.

Bourtzis K, Braig HR (1999). The many faces of Wolbachia. In: Raoult D, Brouqui P (eds) Rickettsiae and Rickettsial Diseases at the Turn of the Third Millennium. Elsevier: Paris, pp 199-219.

Boyle L, O'Neill SL, Robertson HM, Karr TL (1993). Interspecific and intraspecific horizontal transfer of Wolbachia in Drosophila. Science 260: 1796-1799.

Clancy D, Hoffmann AA (1997). Behavior of Wolbachia endosymbionts from Drosophila simulans in Drosophila serrata, a novel host. Am Nat 149: 975-988.

Hurst GD, Johnson AP, Schulenburg JH, Fuyama Y (2000). Male-killing Wolbachia in Drosophila: a temperature-sensitive trait with a threshold bacterial density. Genetics 156: 699-709.

Ikeda T, Ishikawa H, Sasaki T (2003a). Regulation of Wolbachia density in the Mediterranean flour moth, Ephestia kuehniella, and the almond moth, Cadra cautella. Zool Sci 20: 153-157.

Ikeda T, Ishikawa $\mathrm{H}$, Sasaki $\mathrm{T}$ (2003b). Infection density of Wolbachia and level of cytoplasmic incompatibility in the Mediterranean flour moth, Ephestia kuehniella. J Invertebr Pathol 84: 1-5.

Jeyaprakash A, Hoy MA (2000). Long PCR improves Wolbachia DNA amplification: wsp sequences found in $76 \%$ of sixtythree arthropod species. Insect Mol Biol 4: 393-405.

Kittayapong P, Jamnongluk W, Thipaksorn A, Milne JR, Sindhusake C (2003). Wolbachia infection complexity among insects in the tropical rice-field community. Mol Ecol 12: 1049-1060.

Merçot H, Llorente B, Jacques M, Atlan A, Montchamp-Moreau C (1995). Variability within the Seychelles cytoplasmic incompatibility system in Drosophila simulans. Genetics 141: 1015-1023.

Mouton L, Henri H, Bouletreau M, Vavre F (2003). Strainspecific regulation of intracellular Wolbachia density in multiply infected insects. Mol Ecol 12: 3459-3465.

O'Neill SL, Giordano R, Colbert AME, Karr TL, Robertson HM (1992). 16SrRNA phylogenetic analysis of the bacterial endosymbionts associated with cytoplasmic incompatibility in insects. Proc Natl Acad Sci USA 89: 2699-2702.

O'Neill SL, Karr TL (1990). Bidirectional incompatibility between conspecific populations of Drosophila simulans. Nature 348: 178-180.

Perrot-Minnot MJ, Guo LR, Werren JH (1996). Single and double infections with Wolbachia in the parasitic wasp Nasonia vitripennis: effects on compatibility. Genetics 143: 961-972.

Poinsot D, Bourtzis K, Markakis G, Savakis C, Merçot H (1998). Wolbachia transfer from Drosophila melanogaster into $D$. simulans: host effect and cytoplasmic incompatibility relationships. Genetics 150: 227-237. 
Rousset F, Braig HR, O’Neill SL (1999). A stable triple Wolbachia infection in Drosophila with nearly additive incompatibility effects. Heredity 82: 620-627.

Rousset F, De Stordeur E (1994). Properties of Drosophila simulans strains experimentally infected by different clones of the bacterium Wolbachia. Heredity 72: 325331.

Rousset F, Solignac M (1995). Evolution of single and double Wolbachia symbioses during speciation in the Drosophila simulans complex. Proc Natl Acad Sci USA 92: 6389-6393.

Sasaki T, Ishikawa H (1999). Wolbachia infection and cytoplasmic incompatibility in the Almond moth and the Mediterranean flour moth. Zool Sci 16: 739-744.

Sasaki T, Kubo T, Ishikawa H (2002). Interspecific transfer of Wolbachia between two lepidopteran insects expressing cytoplasmic incompatibility: a Wolbachia variant naturally infecting Cadra cautella causes male killing in Ephestia kuehniella. Genetics 162: 1313-1319.

Sinkins SP, Braig HR, O'Neill, SL (1995). Wolbachia pipiens: bacterial density and unidirectional cytoplasmic incompatibility between infected populations of Aedes albopictus. Exp Parasitol 81: 284-291.

Stouthamer R, Breeuwer JAJ, Hurst GDD (1999). Wolbachia pipientis: microbial manipulation of arthropod reproduction. Annu Rev Microbiol 53: 71-102.

Turelli M, Hoffmann AA (1991). Rapid spread of an incompatibility factor among natural Drosophila simulans populations. Nature 353: 440-442.

Werren JH (1997). Biology of Wolbachia. Annu Rev Entomol 42: 587-609.

Werren JH, Zhang W, Guo LR (1995). Evolution and phylogeny of Wolbachia: reproductive parasites of arthropods. Proc $R$ Soc Lond B 261: 55-71. 